\title{
Modeling of free dendritic growth of succinonitrile-acetone alloys with thermosolutal melt convection
}

\author{
Q. Li, C. Beckermann* \\ Department of Mechanical and Industrial Engineering, The University of Iowa, 2412 Seamans Center, Iowa City, IA 52242, USA
}

Received 8 May 2001; accepted 9 November 2001

Communicated by G.B. McFadden

\begin{abstract}
A stagnant film model of the effects of thermosolutal convection on free dendritic growth of alloys is developed, and its predictions are compared to available earth-based experimental data for succinonitrile-acetone alloys. It is found that the convection model gives excellent agreement with the measured dendrite tip velocities and radii for low solute concentrations. However, at higher solute concentrations the present predictions show some deviations from the measured data, and the measured (thermal) Peclet numbers tend to fall even below the predictions from diffusion theory. Furthermore, the measured selection parameter $\sigma^{*}$ is significantly above the expected value of 0.02 and exhibits strong scatter. It is shown that convection is not responsible for these discrepancies. Some of the deviations between the predicted and measured data at higher supercoolings could be caused by measurement difficulties. The systematic disagreement in the selection parameter for higher solute concentrations and all supercoolings examined, indicate that the theory for the selection of the dendrite tip operating state in alloys may need to be reexamined. (C) 2002 Elsevier Science B.V. All rights reserved.
\end{abstract}

PACS: $68.70 .+\mathrm{w} ; 81.30 . \mathrm{Fb}$

Keywords: A1. Convection; A1. Dendrites; A1. Growth models; A1. Segregation; B1. Alloys; B1. Succinonitrile

\section{Introduction}

Equiaxed dendrites are a frequently observed growth mode in metal alloy castings. Unlike columnar growth that occurs in directional solidification into a positive temperature gradient, equiaxed growth occurs within a supercooled melt

\footnotetext{
*Corresponding author. Tel.: +1-319-335-5681; fax: +1319-335-5669.

E-mail address: becker@engineering.uiowa.edu (C. Beckermann).
}

where the crystals grow in an unconstrained or "free" manner until they interact with neighboring crystals or container walls. In free dendritic growth, the latent heat of fusion and the solute are rejected into the liquid ahead of the solidliquid interface and the temperature gradient in the liquid at the interface is negative.

Free dendritic growth in pure substances, where the driving force is purely thermal, has been studied extensively and is reasonably well understood for both diffusion and convection controlled cases [1-8]. The presence of coupled heat and 
species transport significantly complicates the analysis of free dendritic growth in alloys. The velocity of the dendrite tip experiences a maximum at a small, but finite solute concentration. Solute additions destabilize the tip, resulting in a smaller tip radius and hence a larger tip velocity. On the other hand, the much lower diffusion rate of solute compared to heat slows down the growth. These two competing effects cause the velocity maximum at small solute concentrations. Theories that have been proposed for diffusion-controlled free dendritic growth in alloy qualitatively predict the velocity maximum (see below for a review). However, on earth and at low supercoolings, buoyancy driven convection is known to significantly influence the tip velocities and the overall growth of a dendrite [5]. In alloys, such convection is driven by both thermal and solutal gradients in the melt. The effect of convection on the dendritic growth of alloys is not yet fully understood despite its importance in metal alloy casting.

In this paper, a simple model is presented to describe the influence of thermosolutal convection on free dendritic growth of alloys. The model is developed within the framework of the wellknown Lipton, Glicksman and Kurz (LGK) formulation $[9,10]$ for diffusion-controlled growth at low Peclet numbers, with the thermosolutal convection effects incorporated through a stagnant film approximation $[11,12]$. The model was primarily developed to predict quantitatively the dendrite tip velocities and radii measured in the earth-based succinonitrile-acetone (SCN-ACE) experiments of Chopra et al. [13]. To the authors' best knowledge, these remain the only experiments where both the dendrite tip velocity and radius were measured over a sufficiently large range of supercoolings and alloy compositions, and under conditions where convection can be expected to be important. Other experimental studies on dendritic growth in alloys (see, for example, Refs. [14-17]) do not report both the tip velocity and radius. The SCN-ACE system is well suited for comparison with theories because of the availability of a large body of both microgravity (diffusion controlled) and terrestrial (convection controlled) experimental data in the limit of vanishing solute concentration (i.e., pure SCN) $[4,5,18]$, and because all relevant thermophysical properties are known accurately. The data of Chopra et al. [13] displays the velocity maximum at small solute concentrations and has often been used to assess dendritic growth theories (see, for example, the review by Trivedi and Kurz [19]). These comparisons show relatively large discrepancies between the predictions and the measurements, especially at higher solute concentrations. It is shown in this paper that the discrepancies can only partially be explained by the influence of convection in the experiments, and that additional study of the alloy dendritic growth problem may be needed.

The paper is structured as follows. In Section 2, current theories of free dendritic growth of alloys are summarized, while Section 3 reviews previous models for pure substances that take into account the effect of natural convection. The present model of thermosolutal convection in free dendritic growth of alloy dendrites is presented in Section 4. Model results are presented in Section 5 and are compared to experiments for both pure SCN and SCN-ACE alloys. The conclusions of the present study are summarized in Section 6 .

\section{Theories of free dendritic growth of alloys}

Before developing a model that includes convection, it is necessary to briefly review available theories of diffusion-controlled free growth of alloy dendrites into a supercooled melt. These basic theories are concerned with the prediction of the steady-state tip velocity, $V$, and radius, $R$, of a branchless needle crystal. Langer [20], Lipton et al. (LGK) [9,10], and Karma and Langer [21] have proposed the first models of free growth into a supercooled alloy melt. These models all use marginal stability theory [22] to determine the operating point of the tip through the introduction of a stability constant $\sigma^{*} \sim 1 /\left(R^{2} V\right)$. Lipton, Kurz, and Trivedi (LKT) [23] and Boettinger, Coriell, and Trivedi (BCT) [24] generalized the LGK model and extended it to high growth rates. The LGK model (or LKT or BCT models for rapid growth) is now the most commonly used model for free dendritic growth from a supercooled alloy melt and is reviewed in greater detail next. In 1985, 
Karma and Kotliar [25] developed the first microscopic solvability theory (MST) for the alloy case based on a boundary layer approximation for the thermal and solutal diffusion fields. Ben Amar and Pelce [26] generalized this theory to a fully non-local model for alloy dendrites at low supercoolings. The MST theory uses the same framework as the LGK theory, except that the selection parameter $\sigma^{*}$ is a function of the surface energy anisotropy strength.

The dendrite tip velocity and radius can be predicted by first considering the thermal and solutal transport at the tip. Taking into account the capillary correction and neglecting the kinetic effect, the total imposed supercooling is given by

$\Delta T=\Delta T_{\mathrm{T}}+\Delta T_{\mathrm{C}}+\Delta T_{\mathrm{R}}$,

where $\Delta T_{\mathrm{T}}, \Delta T_{\mathrm{C}}$, and $\Delta T_{\mathrm{R}}$ are the thermal, solutal, and capillary contributions to the supercooling, respectively. The dimensionless thermal and solutal supercoolings are defined, respectively, as

$\Omega_{\mathrm{T}}=\frac{T_{\mathrm{t}}^{*}-T_{0}}{L_{\mathrm{f}} / c_{\mathrm{L}}} \quad$ and $\quad \Omega_{\mathrm{C}}=\frac{C_{\mathrm{t}}^{*}-C_{0}}{C_{\mathrm{t}}^{*}(1-k)}$,

where $L_{\mathrm{f}}$ is the latent heat of fusion, $c_{\mathrm{L}}$ is the liquid specific heat, $k$ is the partition coefficient, $T_{0}$ and $C_{0}$ are the initial or far-field melt temperature and solute concentration, respectively, and $T_{\mathrm{t}}^{*}$ and $C_{\mathrm{t}}^{*}$ are the temperature and solute concentration in the liquid at the dendrite tip, respectively. Approximating the capillary correction using the GibbsThomson relation, Eq. (1) can be rewritten as [19]

$\Delta T=\left(\frac{L_{\mathrm{f}}}{c_{\mathrm{L}}}\right) \Omega_{\mathrm{T}}+\frac{k \Delta T_{0} \Omega_{\mathrm{C}}}{1-(1-k) \Omega_{\mathrm{C}}}+\frac{2 \Gamma}{R}$,

where $\Gamma$ is the Gibbs-Thomson coefficient and $\Delta T_{0}=m C_{0}(1-1 / k)$ is the equilibrium freezing temperature range, in which $m$ is the liquidus slope. The capillary contribution in Eq. (3) is relatively small for the supercoolings considered here, but is included nevertheless for completeness. The dimensionless thermal and solutal supercoolings in Eq. (3) are obtained from appropriate solutions of the transport problem for the dendrite tip. In the LGK model for diffusion-controlled growth, they are calculated from the Ivantsov solutions for steady heat and species diffusion around a paraboloid of revolution, which can be written in terms of the thermal $\left(P e_{\mathrm{T}}=V R / 2 \alpha\right)$ and solutal $\left(P e_{\mathrm{C}}=V R / 2 D\right)$ Peclet numbers as

$\Omega_{\mathrm{T}}=I v\left(P e_{\mathrm{T}}\right)$ and $\Omega_{\mathrm{C}}=I v\left(P e_{\mathrm{C}}\right)$,

where $I v$ is the Ivantsov function [19], $D$ is the liquid mass diffusivity, and $\alpha$ is the liquid thermal diffusivity. According to the above equations, if the capillary correction can be neglected, the total supercooling is a function of the thermal and solutal Peclet numbers only. In the presence of convection, Eqs. (4) are obviously not valid.

Introducing the stability constant or selection parameter $\sigma^{*}$, a second equation for the unknown dendrite tip velocity and radius is obtained. As shown by Trivedi and Kurz [19,27,28], for an isothermal solid with no solute diffusion in the solid, this equation can be written in the following general form

$R=\left[\frac{\Gamma}{\sigma^{*}\left(m G_{\mathrm{C}}-G_{\mathrm{T}} / 2\right)}\right]^{1 / 2}$,

where the thermal, $G_{\mathrm{T}}$, and solutal, $G_{\mathrm{C}}$, gradients are given by the following general flux balances at the interface

$G_{\mathrm{T}}=-(V / \alpha) L_{\mathrm{f}} / c_{\mathrm{L}}$,
$G_{\mathrm{C}}=-(V / D) C_{\mathrm{t}}^{*}(1-k)$.

Corrections in Eq. (5) for large Peclet numbers [19] are not needed for the small supercoolings considered here. Substituting Eqs. (6) and (7) into Eq. (5), the following tip radius selection criterion for free dendritic growth of alloys is obtained

$R=\frac{d_{0}}{\sigma^{*}}\left[2 P e_{\mathrm{C}}\left(\frac{k \Delta T_{0} /\left(L_{\mathrm{f}} / c_{\mathrm{L}}\right)}{1-(1-k) \Omega_{\mathrm{C}}}\right)+P e_{\mathrm{T}}\right]^{-1}$,

where $d_{0}=\Gamma /\left(L_{\mathrm{f}} / c_{\mathrm{L}}\right)$ is the capillary length. According to Trivedi and Kurz [19], $\sigma^{*}$ as defined above is constant for a given alloy system (implying a fixed anisotropy strength) and independent of the Peclet numbers and solute concentration. Evidence indicates that for the experiments considered here, $\sigma^{*}$ is also independent of the convection velocities in the melt. This issue is examined in greater detail below. In the LGK model for diffusion-controlled growth, $\Omega_{\mathrm{C}}$ in Eq. (8) is calculated from the Ivantsov solution given by Eq. (4), $\Omega_{\mathrm{C}}=I v\left(P e_{\mathrm{C}}\right)$. As mentioned 
above, a different relation for $\Omega_{\mathrm{C}}$ must be used in the presence of convection.

\section{Models of free dendritic growth of pure substances in the presence of natural convection}

The effects of melt flow on free dendritic growth of pure substances have been investigated both experimentally and analytically for almost three decades. Lee et al. [5] present a comprehensive review of the literature on this subject. While most studies focus only on how heat transport away from the tip is modified by flow, Bouissou and Pelce [29] have extended the linearized solvability theory, which assumes a parabolic tip shape, to make quantitative predictions of the effect of a forced flow on the tip selection, i.e., on $\sigma^{*}$. They found that when the external flow velocity is small, the selection parameter $\sigma^{*}$ is independent of the flow, but after a critical threshold value of the flow velocity the inverse of the selection parameter $1 / \sigma^{*}$ increases almost linearly with the external flow velocity.

The theories that have been developed to predict the heat transport away from the tip in the presence of convection all reduce to the Ivantsov solution, Eq. (4), in the limit of no flow [5]. Ananth and Gill [8] and Canright and Davis [30] derived exact solutions for various limiting cases involving thermally driven natural convection. These solutions are relatively complex and would be difficult to extend to thermosolutal convection in the case of alloys. A more simple approach is afforded by the use of so-called stagnant film models. Cantor and Vogel [11] derived a modified Ivantsov solution where the far-field supercooling condition is not applied at infinity, but at a confocal paraboloid located at a distance $\delta_{\mathrm{T}}$ from the dendrite tip, as

$$
\begin{aligned}
\Omega_{\mathrm{T}}= & P e_{\mathrm{T}} \exp \left(P e_{\mathrm{T}}\right)\left\{E_{\mathrm{I}}\left(P e_{\mathrm{T}}\right)\right. \\
& \left.-E_{\mathrm{I}}\left[P e_{\mathrm{T}}\left(1+2 \delta_{\mathrm{T}} / R\right)\right]\right\},
\end{aligned}
$$

where $E_{\mathrm{I}}$ is the exponential integral function. For $\delta_{\mathrm{T}} / R \rightarrow \infty$, the above equation reduces to the Ivantsov solution. The effects of convection on the growth are incorporated into the stagnant film model by evaluating the thickness of the thermal boundary layer, $\delta_{\mathrm{T}}$. This can be accomplished by choosing a suitable Nusselt number, $N u$, correlation for the convective heat transfer at the dendrite tip and realizing that $\delta_{\mathrm{T}} \sim \ell / N u_{\ell}$, where $\ell$ is a characteristic length scale for convection. Then, the ratio $\delta_{\mathrm{T}} / R$ needed in Eq. (9) is given by

$\delta_{\mathrm{T}} / R \sim(\ell / R) / N u_{\ell}$.

Cantor and Vogel [11] used the dendrite tip radius as the characteristic length scale for convection, i.e. $\ell=R$, so that $\delta_{\mathrm{T}} / R \sim 1 / N u_{\mathrm{R}}$. This implies that a larger or global length scale, such as the size of the entire crystal or the size of the system in which the growth takes place, does not influence the convective heat transfer and, hence, the growth.

Sekerka et al. [12] used the stagnant film model to investigate the effect of orientation of a dendritic crystal relative to gravity on its growth in the presence of thermal natural convection. The characteristic length scale was chosen to be the length, $L$, of the dendrite arm, i.e. $\ell=L$, implying that the convection is driven by the overall size of the crystal. The Nusselt number was evaluated from a correlation for free convection from a sphere of radius $L$, i.e. $N u_{\mathrm{L}} \sim R a_{\mathrm{L}}^{1 / 4}$, where $R a$ is the Rayleigh number. Since $R a_{\mathrm{L}} \sim L^{3}$, Eq. (10) can be rewritten in terms of a Rayleigh number based on the tip radius $\mathrm{R}$ as $\delta_{\mathrm{T}} / R \sim(L / R)^{1 / 4}\left(R a_{\mathrm{R}}\right)^{-1 / 4}$. Hence, in Sekerka et al.'s model the thermal boundary layer thickness increases with $L^{1 / 4}$, or in other words, the heat transfer rate at the dendrite tip decreases with $L^{-1 / 4}$. This would result in a decrease in the tip velocity as the dendrite continues to increase in size, something that has not been observed experimentally. Typically, one would expect an opposite behavior where the natural convection becomes stronger as the crystal increases in size. Sekerka et al. set the length $L$ to a constant of the order of several millimeters and did not investigate variations in the tip velocity. It should be noted also that Sekerka et al. took the same value for $\sigma^{*}$ in diffusion dominated (microgravity) and convection (terrestrial) cases. They obtained good agreement with the pure SCN data from the earth-based experiments of Huang and Glicksman [31].

Based on the results of two-dimensional phasefield simulations, Tonhardt and Amberg [32] 
recently proposed a Nusselt number correlation for a downward growing dendrite tip in the presence of thermal convection. This correlation is based on the concept that the dendrite tip is a small object in a forced flow that is driven by the natural convection on the scale of the entire crystal. Hence, the Nusselt number at the tip is evaluated using a correlation for forced convection from an object of characteristic size $R$, i.e. $N u_{\mathrm{R}} \sim \operatorname{Pr}^{0.4} \operatorname{Re}_{\mathrm{R}}^{1 / 2}$, where $\operatorname{Re}_{\mathrm{R}}=U R / v$ is the Reynolds number based on the tip radius, $v$ is the kinematic viscosity, and $P r$ is the Prandtl number. The velocity $U$ is obtained by assuming that the flow is driven by natural convection on the scale of the entire crystal and by using the thermal convection analogy $R e_{\mathrm{L}} \sim G r_{\mathrm{L}}^{1 / 2}$, where both the Reynolds and Grashof numbers (Gr) are based on the dendrite arm length $L$. This concept leads to $\delta_{\mathrm{T}} / R \sim(R / L)^{1 / 4}\left(R a_{\mathrm{R}}\right)^{-1 / 4}$, implying that the heat transfer rate at the tip increases with $L^{1 / 4}$. This behavior is opposite to that predicted by Sekerka et al.'s model and appears to be more realistic. However, Tonhardt and Amberg's phase-field simulation results show that the tip heat transfer rate varies only with $L^{1 / 8}$. This reflects an even weaker dependence on the crystal size and results in only a minor increase of the tip velocity during growth [32]. Again, no experimental evidence is available to support any variation with $L$.

Schrage [33] also developed a simplified analytical model of dendritic growth of a pure substance under the influence of natural convection. The natural convection is treated as a tip-local process and is simplified by using a control volume technique. Technically, this model is similar to the stagnant film approach. It also gives good agreement with available experimental data for pure SCN.

\section{Model of free dendritic growth of alloys in the presence of thermosolutal convection}

The present model of free dendritic growth of alloys in the presence of thermosolutal convection is based on the LGK model reviewed in Section 2 and the stagnant film modified Ivantsov solution for incorporating the effect of convection, as reviewed in Section 3. The choice of $\sigma^{*}$ in the presence of convection is discussed in the next section. In the case of alloys, both thermal $\left(\delta_{\mathrm{T}}\right)$ and solutal $\left(\delta_{\mathrm{C}}\right)$ boundary layer thicknesses must be introduced to account for the thermosolutal convection effects in the stagnant film model. Thus, the Ivantsov solutions given in Eq. (4) are replaced by

$$
\begin{aligned}
\Omega_{\mathrm{T}}= & P e_{\mathrm{T}} \exp \left(P e_{\mathrm{T}}\right)\left\{E_{\mathrm{I}}\left(P e_{\mathrm{T}}\right)\right. \\
& \left.-E_{\mathrm{I}}\left[P e_{\mathrm{T}}\left(1+2 \delta_{\mathrm{T}} / R\right)\right]\right\}, \\
\Omega_{\mathrm{C}}= & P e_{\mathrm{C}} \exp \left(P e_{\mathrm{C}}\right)\left\{E_{\mathrm{I}}\left(P e_{\mathrm{C}}\right)\right. \\
& \left.-E_{\mathrm{I}}\left[P e_{\mathrm{C}}\left(1+2 \delta_{\mathrm{C}} / R\right)\right]\right\} .
\end{aligned}
$$

The boundary layer thicknesses are estimated from appropriate Nusselt $(\mathrm{Nu})$ and Sherwood (Sh) number correlations as

$\delta_{\mathrm{T}} / \ell=B / N u_{\ell} \quad$ and $\quad \delta_{\mathrm{C}} / \ell=B / S h_{\ell}$,

where $\ell$ is again a characteristic length scale for convection and $B$ is a constant of proportionality.

Note that the same constant $B$ is used in Eq. (13) for both boundary layer thicknesses. This is supported by the results of previous studies reviewed in Gebhart et al. [34], which show that a constant value of $B \approx 2$ is appropriate for both the thermal and solutal boundary layer thicknesses. This value is valid for the following ranges of the governing dimensionless parameters: $P r=0.7-7.0, \quad S c=0.94-100, \quad$ and $N=0.5-2$, where $S c$ is the Schmidt number and $N$ is the buoyancy ratio. The buoyancy ratio is defined as $N=\beta_{\mathrm{C}} \Delta C /\left(\beta_{\mathrm{T}} \Delta T\right)$, where $\beta_{\mathrm{T}}$ and $\beta_{\mathrm{C}}$ are the thermal and solutal expansion coefficients, respectively, and $\Delta T$ and $\Delta C$ are characteristic temperature and concentration differences, respectively. A positive value for $N$ implies that the thermal and solutal buoyancy effects assist each other. It can be verified that the SCN-ACE experiments considered here fall within the above ranges of the dimensionless parameters (see Table 1 and the results presented in the next section). An exact value for $B$ is difficult to specify because of the ambiguity in defining the edge of the boundary layers. However, $B$ is not equal to unity. The final choice for $B$ is discussed below.

Gebhart et al. [34] examined numerous previous studies of external thermosolutal natural 
Table 1

Properties of succinonitrile and succinonitrile-acetone alloys

\begin{tabular}{lllll}
\hline & Symbol & Property & Value & Ref. \\
\hline SCN & $T_{\mathrm{m}}$ & Melting point & $331.233 \mathrm{~K}$ & {$[18]$} \\
& $\alpha$ & Liquid thermal diffusivity & $1.134 \times 10^{5} \mu \mathrm{m}^{2} / \mathrm{s}$ & {$[18]$} \\
& $\Gamma$ & Gibbs-Thomson coefficient & $6.525 \times 10^{-2} \mathrm{~K} \mu \mathrm{m}$ & {$[18]$} \\
& $L_{\mathrm{f}} / c_{\mathrm{L}}$ & Unit supercooling & $23.13 \mathrm{~K}$ & {$[18]$} \\
& $d_{0}$ & Capillary length & $2.821 \times 10^{-3} \mu \mathrm{m}$ & {$[6,8]$} \\
& $P r$ & Prandtl number & 23.1 & \\
& & & & {$[10]$} \\
SCN-ACE & $D$ & Liquid mass diffusivity & $1.27 \times 10^{3} \mu \mathrm{m}^{2} / \mathrm{s}$ & {$[10]$} \\
& $M$ & Liquidus slope & $-2.16 \mathrm{~K} / \mathrm{mol}^{2} \%$ & {$[10]$} \\
& $k$ & Equilibrium partition ratio & $0.103 \mathrm{~mol} \% / \mathrm{mol} \%$ & {$[38]$} \\
& $\beta_{\mathrm{T}}$ & Thermal expansion coefficient & $7.91 \times 10^{-4} / \mathrm{K}$ at $T_{\mathrm{m}}$ & {$[38]$} \\
& $\beta_{\mathrm{C}}$ & Solutal expansion coefficient & $1.68 \times 10^{-3} / \mathrm{mol} \%$ at $T_{\mathrm{m}}\left(\right.$ converted from $\left.2.32 \times 10^{-3} / \mathrm{wt} \%\right)$ &
\end{tabular}

convection from a variety of geometries. They found that available Nusselt and Sherwood number correlations for $N>0$ (assisting flows) can be written in the following general form:

$$
\begin{aligned}
& N u_{\ell}=A\left(R a_{\ell, \mathrm{T}}\right)^{1 / 4}\left(1+\frac{N}{\sqrt{L e}}\right)^{1 / 4}, \\
& S h_{\ell}=A\left(R a_{\ell, \mathrm{C}}\right)^{1 / 4}\left(1+\frac{\sqrt{L e}}{N}\right)^{1 / 4},
\end{aligned}
$$

where $A$ is a constant independent of $L e$ and $N$. The Lewis number is defined as $L e=S c / P r=$ $\alpha / D$. In the present application to dendritic growth, the thermal and solutal Rayleigh numbers are given, respectively, by

$$
\begin{aligned}
& R a_{\ell, \mathrm{T}}=\frac{\beta_{\mathrm{T}} g\left(T_{\mathrm{t}}^{*}-T_{0}\right) \ell^{3}}{\alpha \nu} \\
& \text { and } R a_{\ell, \mathrm{C}}=\frac{\beta_{\mathrm{C}} g\left(C_{\mathrm{t}}^{*}-C_{0}\right) \ell^{3}}{D v}
\end{aligned}
$$

and the buoyancy parameter is given by

$$
N=\frac{\beta_{\mathrm{C}}\left(C_{\mathrm{t}}^{*}-C_{0}\right)}{\beta_{\mathrm{T}}\left(T_{\mathrm{t}}^{*}-T_{0}\right)}
$$

In view of Eqs. (13)-(17), the ratio of the thermal to solutal boundary layer thicknesses is given by $\delta_{\mathrm{T}} / \delta_{\mathrm{C}}=S h_{\ell} / N u_{\ell}=L e^{3 / 8}$. Using the properties for SCN-ACE alloys provided in Table 1, it can be seen that the thermal boundary layer is approximately five times thicker than the solutal boundary layer. It is emphasized that the above correlations are not valid for alloys where the buoyancy ratio $N$ is negative (such as $\mathrm{Al}-\mathrm{Cu}$ ) $[35,36]$ and for liquid metals $(\operatorname{Pr} \ll 1)$. The convection correlations given by Eqs. (14) and (15) do not include the diffusion limit for vanishing Rayleigh numbers. This is done so that after substitution of the correlations into the stagnant film model, the Ivantsov solution is obtained as the diffusion limit.

The correlations given by Eqs. (14) and (15) have been found to be applicable to both vertical plates and spheres [34]. Different geometries simply result in different values for the constant $A$. For example, the experiments of Mathers et al. [35] showed that for spheres $A=0.5$, if the sphere diameter $d$ is chosen as the characteristic length. In the limit of purely thermal convection $(N=0)$, Eq. (14) becomes $N u_{\ell}=A\left(R a_{\ell}\right)^{1 / 4}$. Hence, the value of the constant $A$ may also be estimated from the extensive data and analyses available for purely thermal convection from various geometries. Acrivos [37] developed a boundary layer analysis for laminar natural convection that can be used to find the Nusselt number for any surface of revolution about an axis of symmetry. As part of the present study, this method was applied to a paraboloid of revolution pointing downward. It was found that $A \approx 0.9$ for the local Nusselt number at the stagnation point (with $\ell=R$ ). Since there is presently some uncertainty in the value for $A$ for a dendrite, this issue is examined further below by comparing the predictions of the present model to experimental data for dendritic 
growth. Nonetheless, based on the analysis for a paraboloid of revolution, a value of $A \approx 0.9$ appears to be most appropriate for a downward growing dendrite tip.

The review of the previous convection models for dendritic growth of a pure substance in Section 3 and the discussion of Nusselt number correlations given above indicate that the tip radius $R$ is an adequate characteristic length scale for natural convection from a downward growing dendrite tip. Furthermore, Chopra et al. [13] do not report any temporal variations in the tip velocity in their SCN-ACE experiments, which implies no dependence on the dendrite arm length and provides only steady values of the tip radius and velocity. Using $\ell=R$, Eqs. (14) and (15) can be combined with Eq. (13) to give the following final expressions for the thermal and solutal boundary layer thicknesses for use in the stagnant film model:

$\frac{\delta_{\mathrm{T}}}{R}=\frac{B}{A}\left(R a_{\mathrm{R}, \mathrm{T}}\right)^{-1 / 4}\left(1+\frac{N}{\sqrt{L e}}\right)^{-1 / 4}$,

$\frac{\delta_{\mathrm{C}}}{R}=\frac{B}{A}\left(R a_{\mathrm{R}, \mathrm{C}}\right)^{-1 / 4}\left(1+\frac{\sqrt{L e}}{N}\right)^{-1 / 4}$.

Note that only the ratio $B / A$ appears in the above expressions. Based on the previous discussion, the estimated value of this ratio for a downward growing dendrite tip is $B / A \approx 2.2$. However, due to the uncertainties in both $A$ and $B$, a more definite value of the ratio is determined in the next section by comparing the predictions of the present model with earth-based dendritic growth experiments for pure SCN. Here, it should be kept in mind that the ratio $B / A$ can vary with the orientation of the dendrite with respect to gravity [12].

\section{Results and discussion}

\subsection{Model calibration for pure SCN}

The ratio $B / A$ in the expressions for the boundary layer thicknesses, Eqs. (18) and (19), is determined using the free dendritic growth data for pure SCN listed in Koss et al. [18]. This can be accomplished by comparing measured and predicted Peclet numbers as a function of the imposed supercooling. The present model equations readily reduce to the limiting case of a pure substance by setting the alloy concentration to zero. Eq. (18) for the thermal boundary layer thickness reduces to $\delta_{\mathrm{T}} / R=(B / A) R a_{\mathrm{R}, \mathrm{T}}^{-1 / 4}$. When convection is absent, $R a_{\mathrm{R}, \mathrm{T}} \rightarrow 0$ and $\delta_{\mathrm{T}} / R \rightarrow \infty$. Then, according to Eq. (11), $P e_{\mathrm{T}}$ is a function of the thermal supercooling, $\Omega_{\mathrm{T}}$, only. However, when predicting $P e_{\mathrm{T}}$ as a function of the total supercooling, $\Delta T, P e_{\mathrm{T}}$ also depends on $\sigma^{*}$, because the capillary supercooling is a function of the tip radius $R$ (see Eq. (3)). According to Eq. (8), $\sigma^{*}$ is needed to calculate $R$. Fortunately, the capillary supercooling is relatively small for the $\Delta T$ considered here. In the presence of convection, the predicted $P e_{\mathrm{T}}$ is a stronger function of $\sigma^{*}$, because the thermal boundary layer thickness, $\delta_{\mathrm{T}}$, depends on the tip radius $R$. Hence, even though the variation of $P e_{\mathrm{T}}$ with $\Delta T$ can be used to assess the transport part of dendritic growth theories, a $\sigma^{*}$ dependence remains especially in the presence of convection.

An appropriate value for $\sigma^{*}$ for pure $\mathrm{SCN}$ can be found from Eq. (8), which reduces for a pure substance to

$\sigma^{*}=\frac{2 d_{0} \alpha}{R^{2} V}$.

By substituting the measured dendrite tip radius and velocity data for both the microgravity and earth-based experiments listed in Ref. [18] into the above equation, a value of $\sigma^{*}=0.02$ is found to reasonably well represent the data at all supercoolings. Hence, this measured value for $\sigma^{*}$ is taken here to determine the ratio $B / A$.

Fig. 1 shows a comparison of the measured and predicted thermal Peclet numbers, $P e_{\mathrm{T}}=R V / 2 \alpha$, as a function of the total supercooling. As shown before, excellent agreement is obtained between the diffusion theory and the microgravity data, except for some disagreement at very low supercoolings due to a wall effect [18]. For the present convection model, three different lines are drawn in Fig. 1, corresponding to $B / A=1.75,2.19$, and 2.65 (i.e., $2.19 \pm 20 \%$ ). The line for $B / A=2.19$ represents the best fit of the present model to the terrestrial data, and good agreement is obtained 


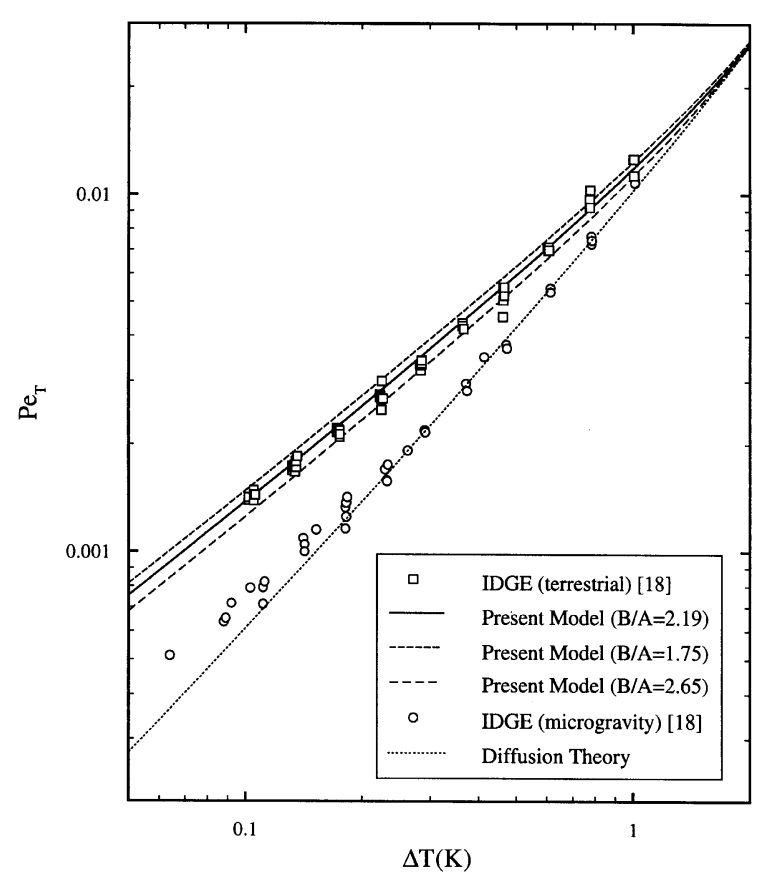

Fig. 1. Comparison of measured and predicted tip Peclet numbers as a function of supercooling for pure SCN.

over the entire supercooling range. The lines for $B / A=1.75$ and 2.65 nicely envelope the terrestrial data, indicating that the uncertainty in $B / A$ is $<20 \%$.

The value $B / A=2.19$ found above is very close to the estimate made in the previous section from an analysis of the thermal convection boundary layer thickness at the stagnation point of a paraboloid of revolution growing downward into the melt (i.e., $B / A \approx 2.2$ ). This agreement lends considerable confidence to the present model, since the terrestrial data of Ref. [18] can be assumed to correspond to downward growing dendrite tips. It also indicates that the choice of the convection length scale $(R)$ is appropriate for pure $\mathrm{SCN}$.

\subsection{Model results for SCN-ACE alloys}

Before comparing the present model for the effect of thermosolutal convection on free dendritic growth to the alloy experiments of Chopra et al. [13], it is instructive to examine the variation of the various model parameters with solute concentration. For this purpose, calculations were per- formed using the properties of SCN-ACE alloys listed in Table $1, B / A=2.19$, and $\sigma^{*}=0.02$. As mentioned in Section $2, \sigma^{*}$ as defined in Eq. (8) should be independent of the solute concentration [19]. Thus, the $\sigma^{*}$ value for pure $\mathrm{SCN}$, as determined from both microgravity and terrestrial data (see Section 5.1), is used in this section. Note that the thermal and solutal expansion coefficients for SCN-ACE alloy melts listed in Table 1 have only recently been measured [38]. The results of the model calculations are shown in Fig. 2.

Fig. 2a shows predicted thermal Peclet numbers, $P e_{\mathrm{T}}$, as a function of solute concentration for three different supercoolings $(\Delta T=0.1,0.5$, and $0.9 \mathrm{~K})$ (note that the solutal Peclet number differs from $P e_{\mathrm{T}}$ only by a constant factor equal to the Lewis number). Corresponding model predictions for diffusion-controlled growth (labeled as "LGK") are included in the figure for easy reference. At vanishing solute concentration $\left(C_{0}=0\right)$, the Peclet numbers in Fig. 2a correspond to those shown in Fig. 1 for pure SCN. With increasing solute concentration, the predicted Peclet numbers decrease for both the convection and the diffusion case. This can be explained by the much lower diffusion rate of solute compared to heat $(\alpha \gg D)$, which tends to reduce the transport at the dendrite tip with increasing $C_{0}$. The Peclet numbers predicted by the convection model are above those from the diffusion theory since in the present model convection always enhances the transport at the tip. As for pure SCN, the predicted convection effect is stronger at the lower supercoolings $(0.1$ and $0.5 \mathrm{~K}$ ) and relatively weak at $0.9 \mathrm{~K}$. Although not readily apparent from the plot in Fig. 2a (due to the log scale used; see also Fig. 6 below), the Peclet numbers predicted by the convection model approach those from the diffusion theory for increasing solute concentration; i.e., the convection effect is weakening with increasing $C_{0}$. As is shown below, this result can be explained by a drastic weakening of the thermal convection for increasing solute concentrations. However, it should not be generalized to solute concentrations $>0.5 \mathrm{~mol} \%$ and alloys other than SCN-ACE.

Fig. $2 b$ shows the calculated variation of the thermal, solutal, and capillary supercoolings with solute concentration for a total supercooling of 

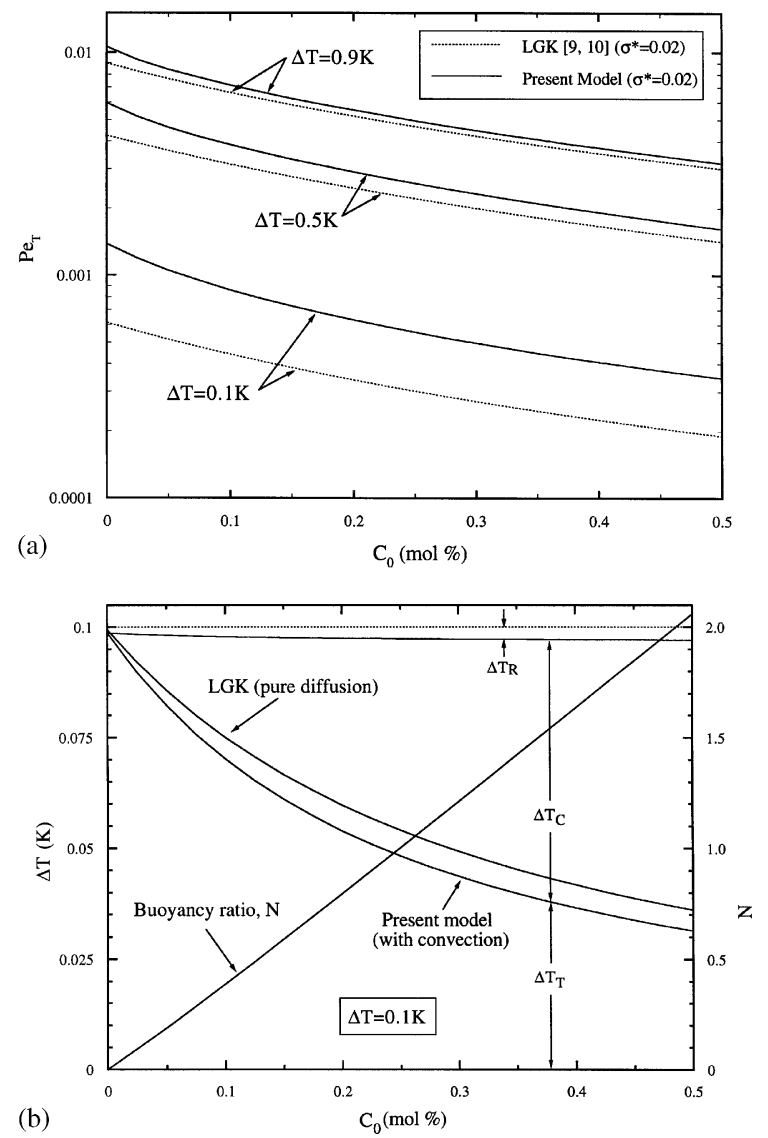

(b)

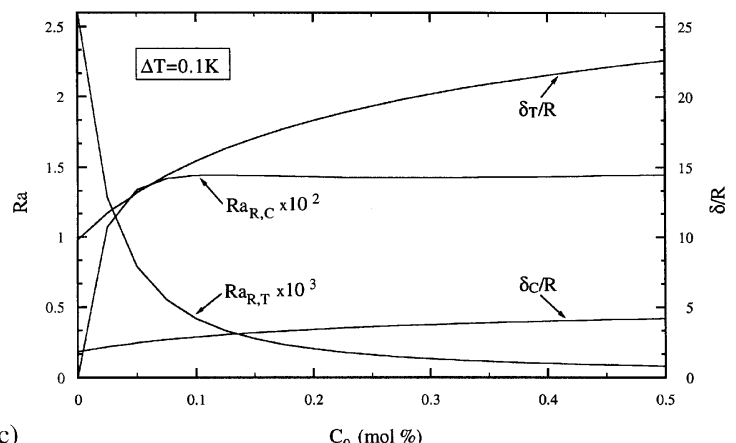

(c)

Fig. 2. Model results for SCN-ACE alloys: (a) variation of predicted tip Peclect numbers with $C_{0}$ for three total supercoolings, (b) variation of predicted thermal, solutal, and capillary supercoolings and the buoyancy ratio with $C_{0}$ for $\Delta T=0.1 \mathrm{~K}$, and (c) variation of predicted Rayleigh numbers and boundary layer thicknesses with $C_{0}$ for $\Delta T=0.1 \mathrm{~K}$.

$\Delta T=0.1 \mathrm{~K}$. With increasing solute concentration, the solutal supercooling increases at the expense of the thermal supercooling, while the capillary contribution remains very small as expected. Interestingly, the differences between the present convection model and the diffusion theory (LGK) are relatively minor, with the convection model giving slightly larger solutal supercoolings. This can be attributed to the fact that regardless of the transport, the three individual supercoolings must add up to the total supercooling ( $0.1 \mathrm{~K}$ in Fig. 2b). The shift between the thermal and solutal contributions to the total supercooling due to convection is simply a reflection of the relative strengths of the thermal and solutal buoyancy forces changing with $C_{0}$. Fig. $2 \mathrm{~b}$ shows that the ratio of the solutal to the thermal buoyancy forces, as described by the parameter $N$, increases almost linearly with $C_{0}$. At the largest solute concentration shown $(0.5 \mathrm{~mol} \%)$, the buoyancy parameter is approximately equal to two.

Fig. 2c shows the calculated variation of the Rayleigh numbers and boundary layer thicknesses with solute concentration for a total supercooling of $\Delta T=0.1 \mathrm{~K}$. The thermal Rayleigh number, $R a_{\mathrm{R}, \mathrm{T}}$, decreases sharply as soon as some solute (ACE) is added to the SCN. This can be attributed to the simultaneous decreases in the thermal supercooling (see Fig. 2b) and the dendrite tip radius, $R$, (see Fig. 3a below) with increasing $C_{0}$. The decrease in $R a_{\mathrm{R}, \mathrm{T}}$ becomes more gradual at solute concentrations greater than about $0.1 \mathrm{~mol} \%$. On the other hand, the solutal Rayleigh number, $R a_{\mathrm{R}, \mathrm{C}}$, increases strongly for solute concentrations below $0.1 \mathrm{~mol} \%$, and then reaches an approximately constant value. This can be explained by the competition between the increasing solutal supercooling and the decreasing tip radius in $R a_{\mathrm{R}, \mathrm{C}}$. Note that $R a_{\mathrm{R}, \mathrm{C}} / R a_{\mathrm{R}, \mathrm{T}}=N L e$. The continued decrease in $R a_{\mathrm{R}, \mathrm{T}}$, coupled with the constancy in $R a_{\mathrm{R}, \mathrm{C}}$, for $C_{0}>0.1 \mathrm{~mol} \%$ explains why the effect of convection on the predicted Peclet numbers (and, hence, the transport at the dendrite tip) diminishes with increasing solute concentration, as noted in connection with Fig. 2a. Finally, Fig. 2c shows that the dimensionless thermal, $\delta_{\mathrm{T}} / R$, and solutal, $\delta_{\mathrm{C}} / R$, boundary layer thicknesses both increase with increasing solute concentration. This also implies that the convection effect is weakening with increasing $C_{0}$. It is interesting to note that the solutal boundary layer 
thickness ranges from one to five times the tip radius for $\Delta T=0.1 \mathrm{~K}$. As noted earlier, $\delta_{\mathrm{T}} / \delta_{\mathrm{C}}=$ $L e^{3 / 8} \approx 5.4$.

\subsection{Comparison of the stagnant film model with $S C N-A C E$ experiments of Chopra et al.}

The present model of free dendritic growth in the presence of thermosolutal convection is compared with the earth-based experiments of Chopra et al. [13] for SCN-ACE alloys. The dendrite tip velocity and radius data were extracted from the figures in Chopra et al. The estimated uncertainty in reading each individual datum is included in the following figures (Figs. 3-5). No uncertainties are shown for the data at $0.5 \mathrm{~K}$, because these data could be read more accurately than the size of the symbols. Chopra et al. do not discuss their experimental uncertainty. Also included in the following figures are some of the terrestrial data for pure SCN from Ref. [18], already shown in Fig. 1. The data from Chopra et al. [13] for $C_{0}=0$ agree well with those from Ref. [18], indicating that the procedure used for extracting the data from the figures in Chopra et al. is relatively accurate. The agreement for $C_{0}=0$ also implies that the data from Refs. [13] and [18] correspond to the same or similar orientations of the dendrite with respect to gravity (i.e., downward growth).

As a first step, the measured and predicted dendrite tip velocities and radii as a function of the solute concentration $C_{0}$ are compared for imposed total supercoolings, $\Delta T$, of $0.1,0.5$ and $0.9 \mathrm{~K}$ in Figs. 3, 4 and 5, respectively. Corresponding model predictions for diffusion-controlled growth (labeled as "LGK") are included in the figures for easy reference. In this comparison, $B / A=2.19$ and the $\sigma^{*}$ value determined for pure SCN (i.e., $\left.\sigma^{*}=0.02\right)$ are used in the model calculations. As noted before, $\sigma^{*}$ as defined in Eq. (8) should be independent of the solute concentration [19]. Furthermore, since the microgravity and terrestrial data for pure SCN in Ref. [18] yield approximately the same $\sigma^{*}$ at all supercoolings (see Section 5.1), it is reasonable to expect that $\sigma^{*}$ is also independent of the convection intensity in the alloy experiments of Chopra et al. [13]. However, a more detailed
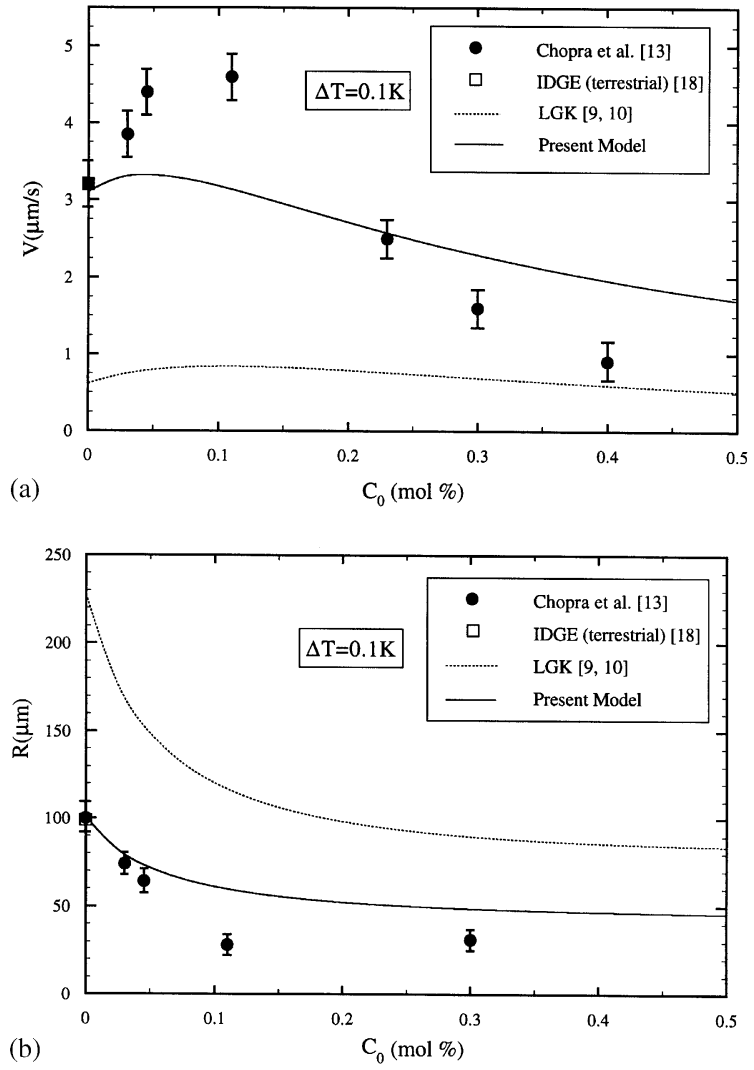

Fig. 3. Comparison of measured and predicted dendrite tip growth velocities (a) and radii (b) as a function of solute concentration for SCN-ACE alloys at $\Delta T=0.1 \mathrm{~K}$.

examination of the effects of solute concentration and convection on $\sigma^{*}$ is provided in Section 5.4.

It can be seen from Figs. 3-5 that there is approximate, qualitative agreement between the predictions and measurements. Both the data and the model show an initial increase in the tip velocity with solute concentration, a maximum around $0.1 \mathrm{~mol} \%$, and a continual decrease at larger $C_{0}$. On the other hand, the tip radii continually decrease with $C_{0}$. As mentioned in the Introduction, the initial strong decrease in the tip radius is caused by destabilization of the tip by the solute, while the decrease in the tip velocity at larger $C_{0}$ is due to the fact the mass diffusivity is much smaller than the thermal diffusivity.

Figs. 3-5 show that the convection effect is strongest at the lowest supercooling. For the $0.1 \mathrm{~K}$ 


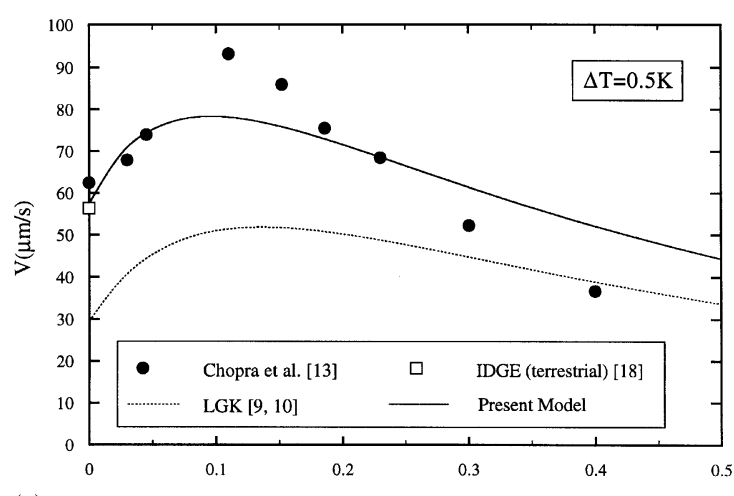

(a)

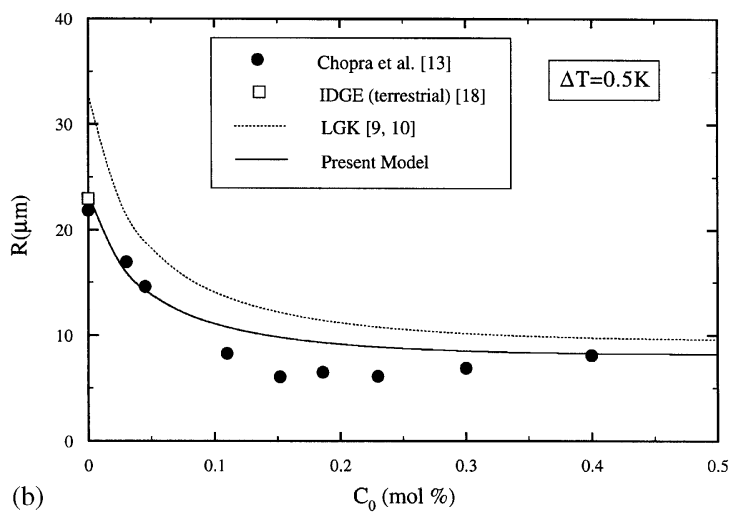

Fig. 4. Comparison of measured and predicted dendrite tip growth velocities (a) and radii (b) as a function of solute concentration for SCN-ACE alloys at $\Delta T=0.5 \mathrm{~K}$.

supercooling (Fig. 3), the maximum measured tip velocity is about four times higher than the prediction from diffusion theory. At the intermediate supercooling $(0.5 \mathrm{~K}$, Fig. 4$)$, the maximum tip velocity is still measured to be about twice the value predicted by the diffusion theory. For both the 0.1 and $0.5 \mathrm{~K}$ supercoolings, the measured tip velocities approach the diffusion theory curve at higher solute concentrations, indicating that the convection effect diminishes as $C_{0}$ increases. At the highest supercooling $(0.9 \mathrm{~K}$, Fig. 5$)$, the present model indicates that the convection effect is small (see also Fig. 2). Surprisingly, except for the datum at $C_{0}=0$, it can be seen from Fig. 5a that the measured tip velocities are, as much as a factor of two, below the curve corresponding to purely diffusive growth. On the other hand, the measured
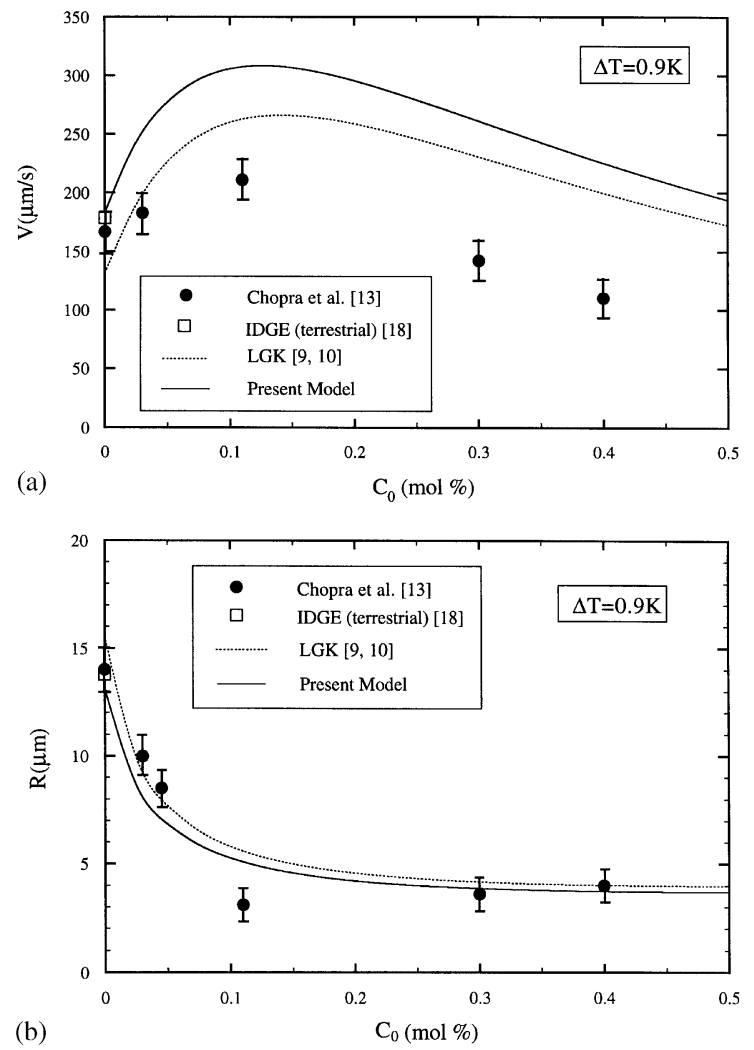

Fig. 5. Comparison of measured and predicted dendrite tip growth velocities (a) and radii (b) as a function of solute concentration for SCN-ACE alloys at $\Delta T=0.9 \mathrm{~K}$.

tip radii at $0.9 \mathrm{~K}$ are relatively close to the predictions.

It is apparent from Figs. 3-5 that the present convection model agrees better with the Chopra et al. data than the diffusion theory. As expected from Fig. 1, the agreement is excellent at vanishing solute concentrations. Overall, the agreement between the measured and predicted tip radii (Figs. 3b-5b) is relatively good at all supercoolings and solute concentrations. However, at higher solute concentrations, large disagreements can be observed in Figs. 3a-5a between the measured and predicted tip velocities. For example, for a supercooling of $0.1 \mathrm{~K}$ (Fig. 3a) when the convection effect is strongest, the measured maximum tip velocity (at around $0.1 \mathrm{~mol} \%$ ) is still $50 \%$ higher than predicted by the present model. At the largest 

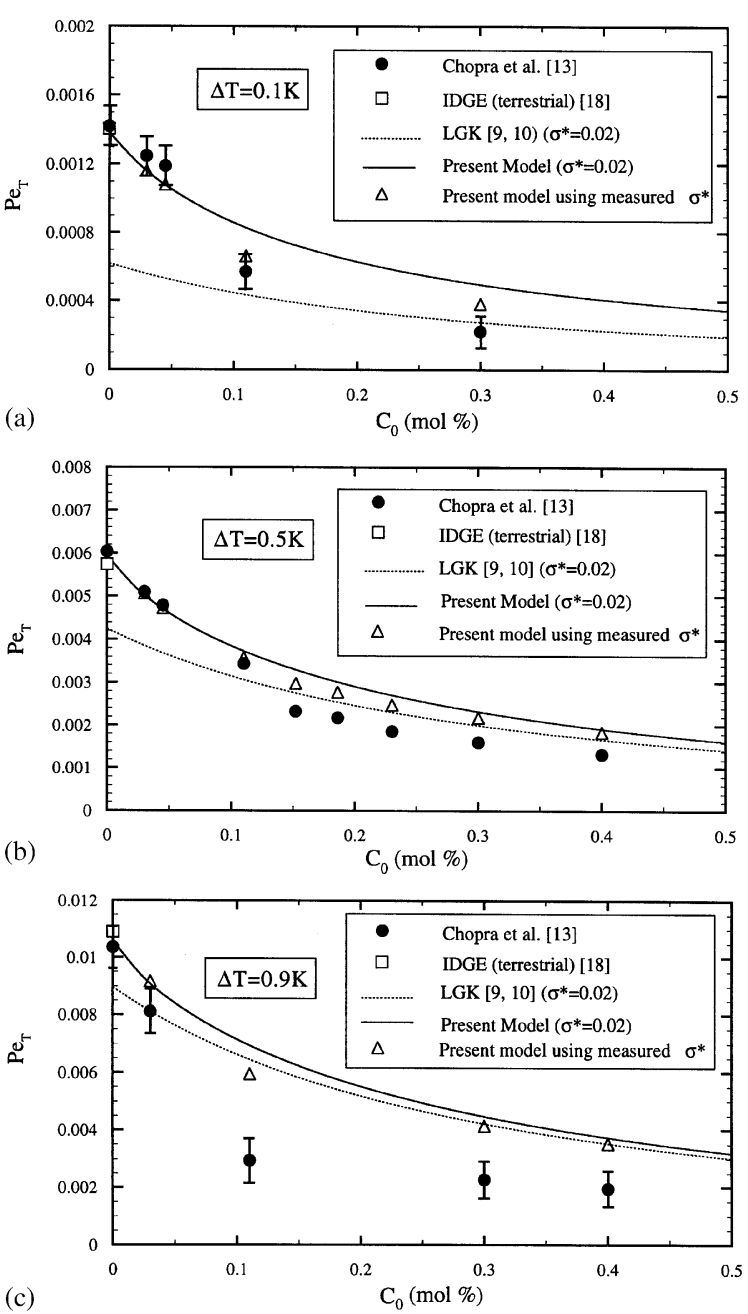

Fig. 6. Comparison of measured and predicted thermal Peclet numbers as a function of solute concentration for SCN-ACE alloys at (a) $\Delta T=0.1 \mathrm{~K}$, (b) $\Delta T=0.5 \mathrm{~K}$, and (c) $\Delta T=0.9 \mathrm{~K}$.

solute concentration $(0.4 \mathrm{~mol} \%)$, the measured velocity is significantly below the prediction from the convection model. At $0.5 \mathrm{~K}$ (Fig. 4), the agreement between the convection model and the measured data appears to be somewhat better, but again the measured tip velocities decrease much faster with increasing solute concentration than predicted. At a supercooling of $0.9 \mathrm{~K}$ (Fig. 5), when the convection effect is least important, the measured velocities at higher solute concentrations are below even the predictions from diffusion theory, and thus the convection model only worsens their prediction.

To better understand the discrepancies between the present convection model and the measured tip velocities at all but the smallest solute concentrations, the measured and predicted thermal Peclet numbers, $P e_{\mathrm{T}}$, are compared in Fig. 6 as a function of solute concentration. It can be seen that at lower solute concentrations (for $C_{0} \leqslant 0.05 \mathrm{~mol} \%$ ), the agreement between the measured and predicted Peclet numbers is good. At higher solute concentrations $\left(C_{0}>0.1 \mathrm{~mol} \%\right)$, however, the experimental $P e_{\mathrm{T}}$ numbers fall consistently below the convection model predictions and at the two higher supercoolings (Figs. 6b and c), they are even below the diffusion predictions.

One possible reason for this behavior could be that the thermosolutal convection reduces the heat transport at the tip, below the diffusion rate. This, in turn, would imply that the melt flows downward along the dendrite, in the same direction as the downward tip velocity, and advects heat/solute from the upstream portion of the dendritic crystal toward the tip. Hence, with increasing solute concentration, the flow would need to reverse direction from upward for pure SCN to downward for more concentrated SCN-ACE alloys. This behavior would be in contradiction with expectation and the present model. Since the buoyancy parameter $N$ is positive in the present model (i.e., the heating of the melt by the dendrite and the rejection of ACE into the melt at the interface both decrease the melt density), the thermal and solutal buoyancy are aiding each other. The flow can be expected to be upward everywhere near the downward growing dendrite tip, because the large $P r$ and $S c$ numbers of the SCN-ACE system imply that the velocity boundary layer is thicker than the thermal and solutal boundary layers.

Another possibility is that the data of Chopra et al. do not correspond to downward growing dendrite tips, but to some other orientation of the dendrite with respect to gravity. For example, if a dendrite were growing upward, the flow would indeed be in the same direction as the tip velocity, resulting in a reduction in the heat/solute transport at the tip. Although Chopra et al. do not explicitly report the directions of the dendrite tip growth 
velocities with respect to gravity, there is strong evidence that all data correspond to approximately downward growing tips [18]. The good agreement between the measured and predicted Peclet numbers at low solute concentrations supports this conclusion. Hence, the orientation effect is unlikely to explain the disagreements in Fig. 6 at higher solute concentrations.

It is especially puzzling that the largest deviations of the measured $P e_{\mathrm{T}}$ from the model occur at a supercooling of $0.9 \mathrm{~K}$ (Fig. 6c), where the convection effect on the growth is weakest. In other words, no matter what the direction of the flow is with respect to the dendrite, one would not expect at this supercooling that the measured $P e_{\mathrm{T}}$ is different from the diffusion value by up to a factor of two.

The above discussion indicates that a different convection model is unlikely to reduce the disagreement between the measured and predicted thermal Peclet numbers at the larger supercoolings and larger solute concentrations. It points to a more fundamental problem with the theory or perhaps the experimental data. Although the dependence of the Peclet number on $\sigma^{*}$ is weak at the larger supercoolings and larger solute concentrations, the use of $\sigma^{*}=0.02$ in the above comparisons also needs to be examined further.

\subsection{Examination of the selection parameter $\sigma^{*}$}

In view of the difficulties in reconciling the experiments and transport theories at higher solute concentrations, it is also useful to examine how the measurements of Chopra et al. [13] agree with the selection criterion in the dendritic growth theory. The general selection criterion for alloys, as reviewed in Trivedi and Kurz [19] and given by Eq. (8), can be solved for the selection parameter $\sigma^{*}$ as

$\sigma^{*}=\frac{d_{0}}{R}\left[2 P e_{\mathrm{C}} \frac{k \Delta T_{0} /\left(L_{\mathrm{f}} / c_{\mathrm{L}}\right)}{1-(1-k) \Omega_{\mathrm{C}}}+P e_{\mathrm{T}}\right]^{-1}$.

It is emphasized again that $\sigma^{*}$ as defined above should be independent of the solute concentration $C_{0}$ and the imposed supercooling [19]. An experimental value for $\sigma^{*}$ can, in principle, be obtained by substituting a measured pair of dendrite tip velocity and radius into Eq. (21) (note that $P e \sim R V)$. However, the presence of the solutal supercooling $\Omega_{\mathrm{C}}$ in Eq. (21) renders $\sigma^{*}$ dependent on the transport at the tip. Note that for pure substances $\Omega_{\mathrm{C}}=0$ and $\sigma^{*}$ is independent of the transport. In the present determination of $\sigma^{*}$ from experimental data, $\Omega_{\mathrm{C}}$ must be calculated, since the solute concentration at the tip, $C_{\mathrm{t}}^{*}$, is not known from measurements. Here, this is accomplished using either the Ivantsov solution given by Eq. (4) or the stagnant film modified Ivantsov solution given by Eq. (12), using measured $V$ and $R$ data.

Fig. 7 shows the variation of the selection parameter $\sigma^{*}$ calculated from the measured tip velocities and radii of Chopra et al. at three supercoolings as a function of the solute concentration $C_{0}$. The uncertainty bars for $\sigma^{*}$ propagate from the uncertainties in the $V$ and $R$ data. It can be seen that all $\sigma^{*}$ obtained using the Ivantsov solution to calculate $\Omega_{\mathrm{C}}$ (labeled as "LGK") are within about $2 \%$ of the $\sigma^{*}$ obtained using the stagnant film model for $\Omega_{\mathrm{C}}$ (labeled as "Present Model"). The difference can be expected to be small, because the solutal supercooling is only weakly affected by convection (see Fig. 2b). Thus, the $\sigma^{*}$ plotted in Fig. 7 are insensitive to the

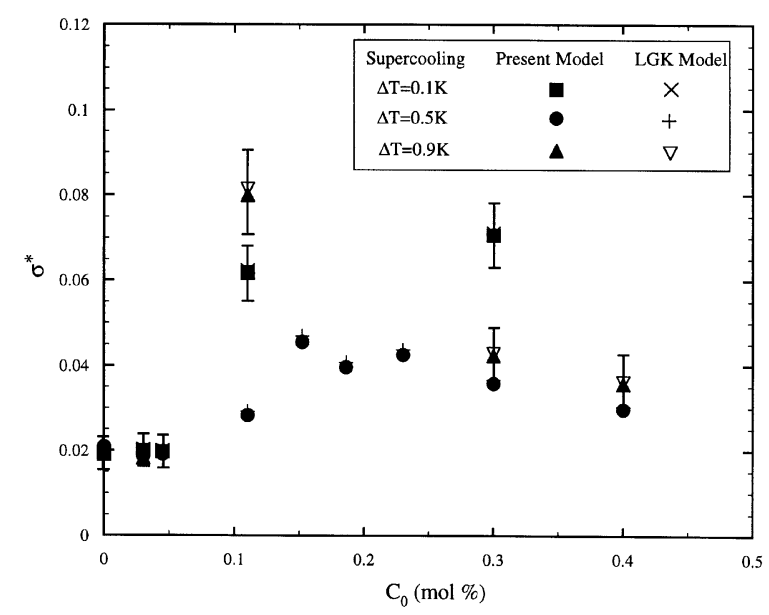

Fig. 7. Selection parameter $\sigma^{*}$ determined from Eq. (21) using the measured dendrite tip velocity and radius data for SCNACE alloys of Chopra et al. [13]. For the symbols labeled "Present Model," the stagnant film model is used to determine $\Omega_{\mathrm{C}}$ in Eq. (21); for the symbols labeled "LGK Model," the Ivantsov model is used for $\Omega_{\mathrm{C}}$. 
convection intensity in the transport solution used to calculate $\Omega_{\mathrm{C}}$; however, this does not necessarily imply that $\sigma^{*}$ is independent of the convection intensity.

Fig. 7 shows that for $C_{0} \leqslant 0.05 \mathrm{~mol} \%, \sigma^{*}$ is indeed independent of the solute concentration. Furthermore, the value of 0.02 determined from the pure SCN microgravity and terrestrial data of Koss et al. [18] is confirmed by the terrestrial Chopra et al. data for $C_{0} \leqslant 0.05 \mathrm{~mol} \%$ and all three supercoolings. Since different supercoolings and solute concentrations imply different convection intensities, it can be said with certainty that $\sigma^{*}$ is indeed independent of the convection intensity for $C_{0} \leqslant 0.05 \mathrm{~mol} \%$.

However, at higher solute concentrations $\left(C_{0}>0.1 \mathrm{~mol} \%\right)$ the experimentally determined $\sigma^{*}$ in Fig. 7 are much larger than 0.02 and can reach values as high as 0.08 . There appears to be no pattern in the variation of $\sigma^{*}$ with the solute concentration. Even if the largest values are ignored, the $\sigma^{*}$ for $C_{0}>0.1 \mathrm{~mol} \%$ still average at least 0.04 . Considering that only the Chopra et al. data appear to be available for direct testing of the free dendritic growth theory for alloys [19], and that this theory has been used literally hundreds of times in a variety of alloy solidification models [39], the lack of agreement at higher solute concentrations is discouraging. Certainly, the scatter in the measured $\sigma^{*}$ for $C_{0}>0.1 \mathrm{~mol} \%$ cannot be explained by any existing theory. As shown before, convection actually becomes weaker with increasing solute concentration; thus, convection is unlikely to be the reason for the scatter in $\sigma^{*}$ for $C_{0}>0.1 \mathrm{~mol} \%$. Furthermore, the convection intensity is primarily controlled by the imposed supercooling, and no consistent variation of the measured $\sigma^{*}$ with $\Delta T$ can be discerned from Fig. 7.

The issue of the dendrite tip operating state selection in SCN-ACE alloys can be viewed from a different perspective by using the following definition of the selection parameter [19]

$\sigma_{\mathrm{C}}^{*}=\frac{2 \alpha d_{0}}{V R^{2}}$.

This alternative definition gives a selection parameter that continually varies with $C_{0}$ but is relatively independent of supercooling. Note that $\sigma_{\mathrm{C}}^{*}=\sigma^{*}$ for $C_{0}=0$. An experimental value of $\sigma_{\mathrm{C}}^{*}$ can simply be obtained by substituting a measured pair of $V$ and $R$ into Eq. (22); thus, the uncertainty with respect to calculation of $\Omega_{\mathrm{C}}$ in Eq. (21) is removed. A prediction of $\sigma_{\mathrm{C}}^{*}$ can be obtained by using the present model to calculate a pair of $V$ and $R$ and then substituting it into Eq. (22).

The measured and predicted $\sigma_{\mathrm{C}}^{*}$ are shown in Fig. 8 as a function of $C_{0}$ for the three supercoolings. The differences between the predictions of the diffusion (labeled as "LGK") and convection (labeled as "Present Model") models are negligibly small, indicating that the convection effect on the calculated $\sigma_{\mathrm{C}}^{*}$ is small. As in the previous figure the agreement between the experiments and theory is excellent for $C_{0} \leqslant 0.05 \mathrm{~mol} \%$. At higher solute concentrations, the measured $\sigma_{\mathrm{C}}^{*}$ values are substantially larger than the predictions. The disagreement appears to be of a systematic nature (as opposed to the scatter in $\sigma^{*}$ in Fig. 7). Note that the differences between Figs. $8 \mathrm{a}-\mathrm{c}$ are minor, indicating that $\sigma_{\mathrm{C}}^{*}$ is relatively independent of the supercooling within the range studied. Hence, the comparison in Fig. 8 indicates again that the disagreement between the measured and predicted $\sigma_{\mathrm{C}}^{*}$ at higher solute concentrations is not due to convection, since the convection intensity is primarily controlled by the imposed supercooling and actually decreases with increasing solute concentration. As with Fig. 7, no explanation for the disagreement is currently available. The systematic nature of the disagreement in Fig. 8 points to some problem with the selection theory for alloys.

\subsection{Effect of the selection parameter $\sigma^{*}$ on the predicted Peclet number}

As shown in Fig. 7, the measured selection parameters $\sigma^{*}$ for higher solute concentrations are significantly different from the 0.02 value used in Section 5.3 (Figs. 3-6) to assess the present convection model. Therefore, it is useful to examine the effect of $\sigma^{*}$ on the predicted thermal Peclet numbers, $P e_{\mathrm{T}}$. As mentioned above, $\sigma^{*}$ affects the heat and solute transport at the dendrite tip through the dependence of the 


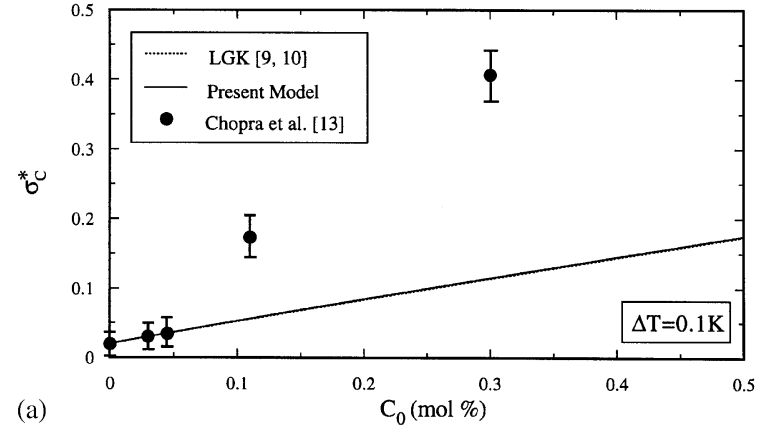

(a)
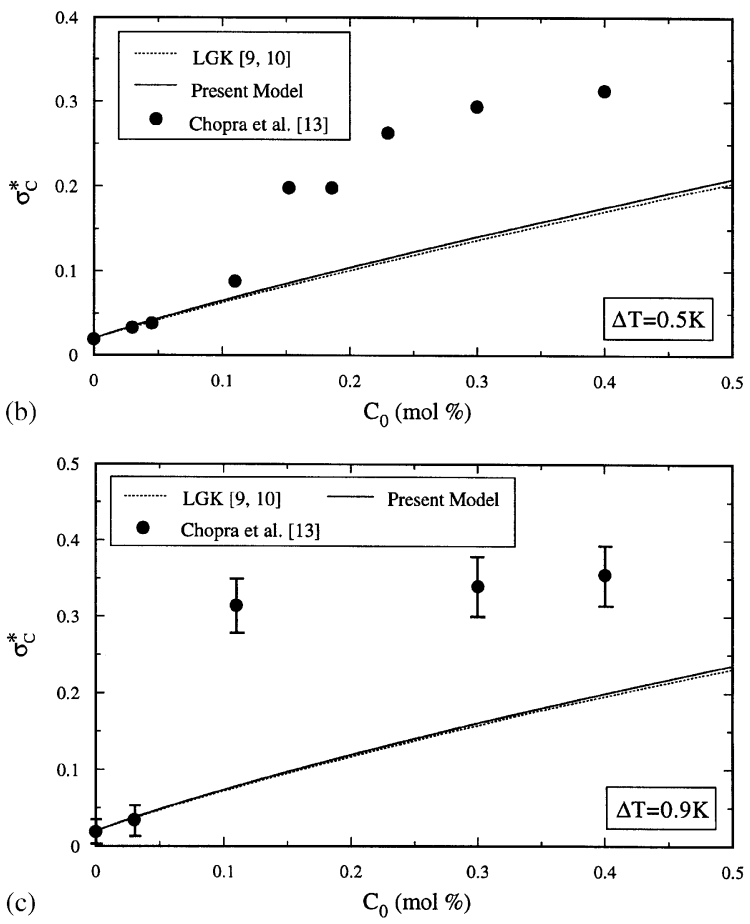

Fig. 8. Comparison of the measured and predicted selection parameter $\sigma_{\mathrm{C}}^{*}$, as defined in Eq. (22), as a function of solute concentration for SCN-ACE alloys at (a) $\Delta T=0.1 \mathrm{~K}$, (b) $\Delta T=0.5 \mathrm{~K}$, and (c) $\Delta T=0.9 \mathrm{~K}$.

convection boundary layer thicknesses on the tip radius and, to a lesser extent, through the capillary correction.

Using the measured $\sigma^{*}$ values from Fig. 7, instead of a constant value of 0.02 , the $P e_{\mathrm{T}}$ numbers are re-calculated with the convection model for each of the data points in the experiments of Chopra et al. These modified predicted
$P e_{\mathrm{T}}$ are included as open triangles in Fig. 6. It can be seen that the predicted $P e_{\mathrm{T}}$ using the measured $\sigma^{*}$ (open triangles) are somewhat closer to the experimental data (solid circles) than the predicted $P e_{\mathrm{T}}$ using $\sigma^{*}=0.02$ (solid line).

At the lowest supercooling of $0.1 \mathrm{~K}$ (Fig. 6a), when the convection effect is strongest, the present model now shows reasonable agreement with the data over almost the entire solute concentration range. Hence, this comparison can be regarded as a validation of the present convection model.

At the two higher supercoolings of 0.5 and $0.9 \mathrm{~K}$ (Figs. $6 \mathrm{~b}$ and c), the use of the measured $\sigma^{*}$ in the convection model still cannot explain the low values of the measured $P e_{\mathrm{T}}$ for higher solute concentrations, although some improvement in the agreement can be noted. Since the convection effect is very small at $0.9 \mathrm{~K}$ and, thus, the influence of $\sigma^{*}$ on $P e_{\mathrm{T}}$ is small, the convection model is unlikely to be the reason for these discrepancies.

\section{Conclusions}

A stagnant film model of thermosolutal convection during free dendritic growth of alloys is developed. The model is based on the standard description of diffusion-controlled free dendritic growth in alloys at low Peclet numbers [19], with the convection effects taken into account through the introduction of thermal and solutal boundary layer thicknesses in the transport solutions for the dendrite tip. The boundary layer thicknesses are evaluated through appropriate Nusselt and Sherwood number correlations for thermosolutal convection that are calibrated using available dendritic growth data for pure SCN. The predictions of the present model are compared to the earth-based experiments of Chopra et al. [13] for SCN-ACE alloys.

It is found that the convection model gives excellent agreement with the measured dendrite tip velocities and radii of Chopra et al. for low solute concentrations. This is significant because the measured tip velocities are up to a factor of four higher than the predictions of the diffusion model, while the measured tip radii are up to a factor of two lower than the diffusion predictions. However, 
at higher solute concentrations the present predictions show some deviations from the measured data, and the measured (thermal) Peclet numbers tend to fall even below the predictions from the diffusion theory. It is particularly puzzling that the largest deviations between the measured and predicted Peclet numbers occur at the highest supercooling $(0.9 \mathrm{~K})$ where the convection effect is very small. Thus, the convection model is unlikely to be the reason for these discrepancies at higher solute concentrations. It is also shown that the use of the measured selection parameter $\sigma^{*}$, instead of a constant value equal to 0.02 , leads to some improvement of the prediction of the measured Peclet numbers, particularly at the lowest supercooling when the convection effect is strongest.

The present comparison of the theories with the experimental data of Chopra et al. also shows that at solute concentrations above $0.1 \mathrm{~mol} \% \mathrm{ACE}$, the measured selection parameter $\sigma^{*}$ is significantly above the expected value of 0.02 and exhibits strong scatter. Similarly, the selection parameter $\sigma_{\mathrm{C}}^{*}$, defined in Eq. (22), is systematically above the theoretical prediction at large solute concentrations. Convection is not responsible for these discrepancies, primarily because the disagreements are independent of the supercooling and because the convection effect becomes weaker with increasing solute concentration.

The above comparisons show that, despite the overall success of the present convection model, available free dendritic growth theories cannot accurately predict all measured dendrite tip velocities and radii in the SCN-ACE experiments of Chopra et al. The persistent disagreements at the highest supercooling $(0.9 \mathrm{~K})$ could be caused by experimental difficulties, since the high tip velocities and very small tip radii (of the order of $5 \mu \mathrm{m})$ in this regime can lead to a large measurement uncertainty [18]. On the other hand, the systematic discrepancies in the selection parameters for higher solute concentrations, even at the lowest supercooling $(0.1 \mathrm{~K})$ where the tip radii are relatively large (of the order of 100 microns), is most likely not due to measurement uncertainties. They indicate that the theory for the selection of the dendrite tip operating state in alloys may need to be reexamined.

\section{Acknowledgements}

This work was supported by NASA under contract NCC8-199.

\section{References}

[1] M.E. Glicksman, S.P. Marsh, in: D.T.J. Hurle (Ed.), Handbook of Crystal Growth, Vol. 1, Elsevier Science Publishers, Amsterdam, 1993, p. 1077.

[2] J.S. Langer, Science 243 (1989) 1150.

[3] J.S. Langer, Rev. Mod. Phys. 52 (1980) 1.

[4] M.E. Glicksman, M.B. Koss, E.A. Winsa, Phys. Rev. Lett. 73 (1993) 573.

[5] Y.-W. Lee, R.N. Smith, M. Glicksman, M.B. Koss, Ann. Rev. Heat Transfer 7 (1997) 59.

[6] R. Ananth, W.N. Gill, J. Crystal Growth 108 (1991) 173.

[7] D.A. Saville, P.J. Beaghton, Phys. Rev. A 37 (1988) 3423.

[8] R. Ananth, W.N. Gill, J. Crystal Growth 91 (1988) 587.

[9] J. Lipton, M.E. Glicksman, W. Kurz, Mater. Sci. Eng. 65 (1984) 57.

[10] J. Lipton, M.E. Glicksman, W. Kurz, Metall. Trans. A 18A (1987) 341.

[11] B. Cantor, A. Vogel, J. Crystal Growth 41 (1977) 109.

[12] R.F. Sekerka, S.R. Coriell, G.B. McFadden, J. Crystal Growth 154 (1995) 370.

[13] M.A. Chopra, M.E. Glicksman, N.B. Singh, Metall. Trans. A 19A (1988) 3087.

[14] M. Kahlweit, J. Crystal Growth 6 (1970) 125.

[15] S.-K. Chan, H.-H. Reimer, M. Kahlweit, J. Crystal Growth 43 (1978) 229.

[16] C.S. Lindenmeyer, G.T. Orrok, K.A. Jackson, B. Chalmers, J. Chem. Phys. 27 (1957) 822.

[17] G.T. Orrok, Ph.D. Thesis, Harvard University, Cambridge, MA, 1958.

[18] M.B. Koss, J.C. LaCombe, L.A. Tennenhouse, M.E. Glicksman, E.A. Winsa, Metall. Mater. Trans. A 30A (1999) 3177.

[19] R. Trivedi, W. Kurz, Inter. Mater. Rev. 39 (1994) 49.

[20] J.S. Langer, Physicochem. Hydrodyn. 1 (1980) 41.

[21] A. Karma, J.S. Langer, Phys. Rev. A 30 (1984) 3147.

[22] J.S. Langer, H. Muller-Krumbhaar, Acta Metall. 26 (1978) 1681.

[23] J. Lipton, W. Kurz, R. Trivedi, Acta Metall. 35 (1987) 957.

[24] W.J. Boettinger, S.R. Coriell, R. Trivedi, in: R. Mehrabian, P.A. Parrish (Eds.), Rapid Solidification Processing: Principles and Technologies, Claitor's Publishing, Baton Rouge, LA, 1985, p. 13.

[25] A. Karma, B.G. Kotliar, Phys. Rev. A 31 (1985) 3266.

[26] M. Ben Amar, P. Pelce, Phys. Rev. A 39 (1989) 4263.

[27] R. Trivedi, J. Crystal Growth 73 (1985) 289.

[28] W. Kurz, R. Trivedi, Acta Metall. 38 (1990) 1.

[29] Ph. Bouissou, P. Pelce, Phys. Rev. A 40 (1989) 6673.

[30] D. Canright, S.H. Davis, J. Crystal Growth 114 (1991) 153. 
[31] S.C. Huang, M.E. Glicksman, Acta Met. 29 (1981) 701.

[32] R. Tonhardt, G. Amberg, Phys. Rev. E 62 (2000) 828.

[33] D.S. Schrage, J. Crystal Growth 205 (1999) 410.

[34] B. Gebhart, Y. Jaluria, R. Mahajan, B. Sammakia, Buoyancy-Induced Flows and Transport, Hemisphere, New York, 1988, p. 310.

[35] W.G. Mathers, A.J. Madden, E.L. Piret, Ind. Eng. Chem. 49 (1957) 961.
[36] J.A. Den Bouter, B. DeMunnik, P.M. Heertjes, Chem. Eng. Sci. 23 (1968) 1185.

[37] A. Acrivos, A.I.Ch.E. J. 6 (1960) 584.

[38] D.L. Ceynar, C. Beckermann, J. Crystal Growth 222 (2001) 380.

[39] M. Rappaz, Int. Mater. Rev. 34 (1989) 93. 